\title{
Mass Analyzer
}

National Cancer Institute

\section{Source}

National Cancer Institute. Mass Analyzer. NCI Thesaurus. Code C47926.

A device that separates a mixture of ions by their mass-to-charge ratios. 